\title{
Diagnosis of nosocomial pneumonia in medical ward: repeatability of the protected specimen brush
}

\author{
B. Herer*,\#, C. Fuhrman*, D. Demontrond*, Z. Gazevic*, B. Housset", C. Chouaïd ${ }^{\#}$
}

Diagnosis of nosocomial pneumonia in medical ward: repeatability of the protected specimen brush. B. Herer, C. Fuhrman, D. Demontrond, Z. Gazevic, B. Housset, C. Chouaï. (C) ERS Journals Ltd 2001.

ABSTRACT: The aims of this study were to assess the repeatability of two pairs of protected specimen brushes (PSB) done successively in the same lung area and either processed at the bedside or in the laboratory, and to provide a description of the bacteriological findings in 39 cases of suspected nosocomial pneumonia occurring in nonventilated patients.

Four PSB were divided into two pairs. One pair of brushes $(\mathrm{PB})$ was prepared at bedside and then sent to the laboratory; the other pair (PL) was immediately sent to the laboratory for complete processing.

According to a $10^{3}$ colony forming units (cfu) $\mathrm{mL}^{-1}$ threshold, $49 \%$ out of $156 \mathrm{PSB}$ were positive. Using the $10^{3} \mathrm{cfu} \cdot \mathrm{mL}$ threshold, the PL brushes were $89.7 \%$ concordant while the $\mathrm{PB}$ brushes were $76.9 \%$ concordant. The repeatability as expressed by K-value of the cultures of PSB was higher for PL brushes than for PB brushes (K-values of 0.795 and 0.537 respectively, $p=0.12$ ). Bacterial species were isolated in $58.3 \%$ of 156 PSB (176 isolates). In 14 cases, cultures of PSB disclosed more than one micro-organism in a concentration $>10^{3} \mathrm{cfu} \cdot \mathrm{mL}^{-1}$. The most frequently isolated organisms were Pseudomonas spp. (23.9\%), Enterobacteriaceae (23.3\%), Streptococcus spp. $(21.6 \%)$ and Staphylococcus spp. (13.1\%). Polymicrobial cultures were more frequent if the patient had a tracheostomy (seven out of the nine patients with a tracheostomy versus seven out of the 30 patients without a tracheostomy, $p<0.01)$. Bacteriological discrepancies leading to a potential troublesome choice in antibiotherapy were observed in $31.8 \%$ of the patients for $P_{L}$ brushes and $56.5 \%$ of the patients for $P_{B}$ brushes.

There is a low degree of repeatability of protected specimen brushes outside intensive care units which seem dependent on sampling processing. The distribution of pathogens found in case of suspicion of nosocomial pneumonia in nonventilated patients appears to be similar to that obtained in ventilator-associated pneumonia. Eur Respir J 2001; 18: 157-163.
*Centre Médical de Forcilles, F-77170 Férolles-Attilly, and ${ }^{\#}$ Centre Hospitalier Intercommunal de Créteil, F-94000 Créteil, France.

Correspondence: B. Herer Centre Médical de Forcilles F-77170 Férolles-Attilly France

Fax: 33164055591

Keywords: Bronchoscopy nosocomial pneumonia reproducibility of results

Received: November 221999 Accepted after revision Janurary 10 2001

Supported in part by a grant from the Association "Compliance".
Nosocomial pneumonia (NP) is the second most common cause of nosocomial infections in France [1]. Mechanical ventilation is a major risk factor for NP [2], and ventilator-associated pneumonia (VAP) may increase the risk of death in critically ill patients [3, 4]. However, less data is available for NP occurring in nonventilated patients than in ventilated patients [5].

In VAP, diagnostic strategy remains controversial $[6,7]$. Even if no diagnostic criteria for NP is totally accurate, protected specimen brush (PSB) or bronchoalveolar lavage (BAL) are the main diagnostic tools. PSB was first described by WIMBERLEY et al. [8]. The transection of the PSB has been reported to be performed either at bedside [9-11] or in the laboratory $[12,13]$, where all the microbiological processing will be finally done. It may be assumed that differences in this procedure may influence the microbiological results.

The aims of this study were to assess the repeatability of two pairs of PSB performed successively in the same lung area, PSB in nonventilated patients, and either processed at the bedside or in the laboratory, and to collect the bacteriological data in 39 cases of suspected NP occurring in nonventilated patients.

\section{Methods}

\section{Patient population}

The Forcilles centre is a 408-bed facility mainly $(70 \%)$ constituted of intermediary care or convalescent beds.

Thirty-nine patients were prospectively and consecutively included in this monocentric study. NP was suspected on relatively wide criteria since clinical criteria alone might not reliably predict patients with and without NP [14]. The following items were required to include patients in the study $[1,3]$ : recent 
and persistent infiltrate on the chest radiograph and 2 of the following items: temperature $\geqslant 38.5^{\circ} \mathrm{C}$ or $<36.5^{\circ} \mathrm{C}$; leukocytosis (white blood cell (WBC) $>15,000 \cdot \mathrm{mm}^{-3}$ ) or leukopenia (WBC $<4,000 \cdot \mathrm{mm}^{-3}$ ); purulent tracheal secretions. No significant microbiological correlations were obtained in this study with blood or pleural fluid cultures, which were all negative; this fact is relevant, with previous reports stating that positive blood [15] or pleural [14] cultures are uncommon in patients with NP. No autopsy specimens were sent for cultures, since histological confirmation [12] of pneumonia is unlikely to be performed in most nonintensive care units. In all patients either the symptoms developed after $72 \mathrm{~h}$ of hospitalization, or the admission in the hospital followed a previous prolonged stay in another hospital. The use of antibiotics within the two previous days was recorded. Each patient gave informed consent to the study.

\section{Study design}

Fibreoptic bronchoscopy was performed with topical anesthaesia using xylocaine limited to the nose and pharynx, except for the nine patients with tracheostomy, for whom no topical anesthaesia was used.

PSB was performed as previously described by Wimberley et al. [8]. Four plugged telescoping catheter brushes were successively inserted through the working channel, advanced $2-3 \mathrm{~cm}$ beyond the tip of the bronchoscope. The distal plug was ejected and the brush was then advanced beyond the tip of the inner cannula $3-4 \mathrm{~cm}$ into the bronchus, gently rotated a few times and retracted a few $\mathrm{cm}$ into the inner cannula. The whole unit was removed from the bronchoscope. The sampling procedure lasted $\sim 5 \mathrm{~min}$. All four PSB were obtained from the same segment or subsegment of the lung.

The PSB were processed in two pairs. For one pair $(\mathrm{PB})$, the processing began outside the laboratory, at the bedside. After cleaning of the distal portion of the catheter with $70 \%$ ethanol, the two brushes ( $\mathrm{PB} 1$ and $\mathrm{P}_{\mathrm{B} 2}$ ). were aseptically cut and placed in a sterile vial containing $1 \mathrm{~mL}$ of lactate Ringer's solution [16] and then sent to the laboratory. For the other pair of brushes (PL), both brushes ( $\mathrm{P}_{L 1}$, and $\mathrm{P}_{2}$ ) were put into a sterile bag and the total processing, including the initial cutting of the brushes, was carried out inside the laboratory. The order of processing $\mathrm{P}_{\mathrm{B}}$ and $P_{L}$ was randomly assigned. All specimens were transported to the laboratory within $30 \mathrm{~min}$. Microbiological procedures were performed for all four brushes according to the protocol described by WIMBERLEY et al. [8]. All vials containing the PSB and medium transport were vortexed. Three 10 -fold dilutions were prepared, and $0.1-\mathrm{mL}$ aliquots of each sample were streaked on four agar plates. Cultures were incubated at $37^{\circ} \mathrm{C}$ under aerobic conditions and in a carbon dioxide $\left(\mathrm{CO}_{2}\right)$ atmosphere. If the culture was positive, counts of colony forming units (cfu) per millilitre, identification of each species, and test of susceptibility to antibiotics were performed. To separate isolates considered to be in high concentration (i.e. confirmed lung infection) from isolates in low concentration (i.e. airway colonization), the previously described cut-off point of $10^{3} \mathrm{cfu} \cdot \mathrm{mL}^{-1}$ was used $[8,12]$.

\section{Statistical methods}

Quantitative data are expressed as mean \pm SD. Chisquared statistics were used for categorical variables. Repeatability of each PSB processing ( $\mathrm{PB}_{\mathrm{B}}$ and $\mathrm{PL}_{\mathrm{L}}$ ) was assessed using $\mathrm{K}$ test. Absence of systematic bias was determined using the MacNemar test. K-values were given with positive and negative (Ppos and Pneg) agreements [17-20].

\section{Results}

Thirty-nine episodes of suspected NP occurring in 39 patients were analysed. Patient characteristics are reported in table 1 . Reasons for hospitalization were: neoplastic disease (oesophagus, larynx and bronchi, $\mathrm{n}=17)$; respiratory insufficiency $(\mathrm{n}=9)$; management of postoperative complications of abdominal surgery $(n=8)$; neurological disease $(n=4)$; cardiac insufficiency $(n=1)$. All patients had at least one risk factor for NP [21]: central nervous system dysfunction, chronic obstructive pulmonary disease (COPD), recent thoracic or abdominal surgery, history of aspiration, age $>65$. Nine patients had a tracheostomy (patients Nos. 9, 16, $22,24,26,29,31,37$ and 38, table 2), and three were ventilated with nocturnal intermittent positive pressure ventilation (IPPV). Nine patients received antibiotics within two days before PSB procedure. Six $(14.6 \%)$ episodes were eventually lethal. All six deaths were attributed to NP. Death occurred in $2 / 3$ ventilated patients versus $4 / 36$ of the nonventilated patients $(\mathrm{p}<0.01)$. No deaths occurred during bronchoscopy. Transient decreased oxygen saturation below $95 \%$ was corrected by additional oxygen. There was no episode of bronchial haemorrhage during bronchoscopy. Using the $10^{3} \mathrm{cfu} \cdot \mathrm{mL}^{-1}$ threshold, PSB culture was positive in 19, 18, 21 and 19 out of 39 patients, for $\mathrm{P}_{\mathrm{B} 1}, \mathrm{~PB}_{\mathrm{B}}, \mathrm{P}_{\mathrm{L} 1}$ and $\mathrm{P}_{\mathrm{L} 2}$ respectively (49\% out

Table 1.-Patients characteristics

\section{Patients characteristics}

\begin{tabular}{lc}
\hline Sex M:F & $25: 14$ \\
Age yrs* & $63.5 \pm 12.7$ \\
Underlying risk factor for NP & \\
Recent surgery & $23(59)$ \\
History of aspiration & $18(46)$ \\
COPD & $17(44)$ \\
Age $>65$ yrs & $15(38)$ \\
CNS dysfunction & $9(23)$ \\
Tracheostomy & $9(23)$ \\
Nocturnal IPPV & $3(8)$ \\
\hline
\end{tabular}

Data presented as n (\%) unless otherwise stated. M: male; F: female; NP: nosocomial pneumonia; COPD: Chronic obstructive pulmonary disease; CNS: central nervous system; IPPV: intermittent positive pressure ventilation. *: data presented as mean \pm SD. 
Table 2. - Clinical outcome, prior antibiotic treatment and culture results of four protected specimen brushes (PSB) according to a $10^{3} \mathrm{cfu} \cdot \mathrm{mL}^{-1}$ threshold in 39 patients

\begin{tabular}{|c|c|c|c|c|c|c|c|}
\hline \multirow{2}{*}{$\begin{array}{l}\text { Patient } \\
\text { No. }\end{array}$} & \multirow[t]{2}{*}{ Clinical outcome } & \multirow{2}{*}{$\begin{array}{l}\text { Prior } \\
\text { antibiotic } \\
\text { treatment }\end{array}$} & \multirow[t]{2}{*}{ Bacterial species } & \multicolumn{2}{|c|}{$\mathrm{PL}_{\mathrm{L}}$} & \multicolumn{2}{|c|}{$\mathrm{PB}$} \\
\hline & & & & $1 \mathrm{st}$ & 2nd & $1 \mathrm{st}$ & $2 \mathrm{nd}$ \\
\hline $\begin{array}{l}1 \\
2 \\
3\end{array}$ & $\begin{array}{l}\text { Deceased } \\
\text { Survived } \\
\text { Survived }\end{array}$ & $\begin{array}{l}\mathrm{N} \\
\mathrm{N} \\
\mathrm{N}\end{array}$ & $\begin{array}{l}\text { P. aeruginosa } \\
\text { nil } \\
\text { nil }\end{array}$ & $\geqslant 10^{3}$ & $\geqslant 10^{3}$ & $\geqslant 10^{3}$ & $\geqslant 10^{3}$ \\
\hline 4 & Deceased & $\mathrm{N}$ & $\begin{array}{c}\text { E. coli } \\
\text { S. epidermidis } \\
\text { Streptococcus spp. }\end{array}$ & $\geqslant 10^{3}$ & $\begin{array}{l}\geqslant 10^{3} \\
\geqslant 10^{3} \\
\geqslant 10^{3}\end{array}$ & $\begin{array}{l}\geqslant 10^{3} \\
\geqslant 10^{3}\end{array}$ & $\geqslant 10^{3}$ \\
\hline $\begin{array}{l}5 \\
6 \\
7\end{array}$ & $\begin{array}{l}\text { Survived } \\
\text { Survived } \\
\text { Survived }\end{array}$ & $\begin{array}{l}\mathrm{Y} \\
\mathrm{N} \\
\mathrm{N}\end{array}$ & $\begin{array}{l}\text { S. aureus } \\
\text { nil } \\
\text { nil }\end{array}$ & $<10^{3}$ & $<10^{3}$ & $\geqslant 10^{3}$ & $\geqslant 10^{3}$ \\
\hline 8 & Survived & Y & Streptococcus spp. & & & & $\geqslant 10^{3}$ \\
\hline 9 & Survived & $\mathrm{N}$ & $\begin{array}{l}\text { A. baumanii } \\
\text { S. epidermidis }\end{array}$ & $\begin{array}{l}\geqslant 10^{3} \\
<10^{3}\end{array}$ & $\geqslant 10^{3}$ & $\geqslant 10^{3}$ & $\geqslant 10^{3}$ \\
\hline 10 & Survived & $\mathrm{N}$ & nil & & & & \\
\hline $\begin{array}{l}11 \\
12 \\
13\end{array}$ & $\begin{array}{l}\text { Survived } \\
\text { Survived } \\
\text { Survived }\end{array}$ & $\begin{array}{l}\mathrm{N} \\
\mathrm{N} \\
\mathrm{Y}\end{array}$ & $\begin{array}{l}\text { H. influenzae } \\
\text { nil } \\
\text { nil }\end{array}$ & $\geqslant 10^{3}$ & $\geqslant 10^{3}$ & $\geqslant 10^{3}$ & $\geqslant 10^{3}$ \\
\hline 14 & Survived & $\mathrm{N}$ & $\begin{array}{c}\text { S. aureus } \\
\text { Streptococcus spp. }\end{array}$ & $<10^{3}$ & & $\begin{array}{l}<10^{3} \\
\geqslant 10^{3}\end{array}$ & \\
\hline 15 & Survived & $\mathrm{N}$ & $\begin{array}{c}\text { S. aureus } \\
\text { P. aeruginosa } \\
\text { Providencia } \mathrm{spp} .\end{array}$ & $\begin{array}{l}\geqslant 10^{3} \\
\geqslant 10^{3} \\
\geqslant 10^{3}\end{array}$ & $\begin{array}{l}\geqslant 10^{3} \\
\geqslant 10^{3} \\
\geqslant 10^{3}\end{array}$ & $<10^{3}$ & $\begin{array}{l}\geqslant 10^{3} \\
\geqslant 10^{3} \\
\geqslant 10^{3}\end{array}$ \\
\hline 16 & Survived & $\mathrm{N}$ & $\begin{array}{l}\text { S. pneumoniae } \\
\text { Moraxella spp. }\end{array}$ & & $\begin{array}{l}\geqslant 10 \\
\geqslant 10^{3} \\
\geqslant 10^{3}\end{array}$ & & $\begin{array}{l}\geqslant 10^{3} \\
<10^{3}\end{array}$ \\
\hline 17 & Survived & $\mathrm{Y}$ & Streptococcus spp. & $\geqslant 10^{3}$ & & & \\
\hline 18 & Deceased & $\mathrm{N}$ & $\begin{array}{l}\text { Streptococcus spp. } \\
\text { Moraxella spp. } \\
\text { Neisseria spp. } \\
\text { H. parainfluenzae }\end{array}$ & $\begin{array}{l}\geqslant 10^{3} \\
\geqslant 10^{3} \\
<10^{3} \\
\geqslant 10^{3}\end{array}$ & $\begin{array}{l}\geqslant 10^{3} \\
\geqslant 10^{3} \\
<10^{3} \\
\geqslant 10^{3}\end{array}$ & & $\begin{array}{l}\geqslant 10^{3} \\
\geqslant 10^{3} \\
<10^{3}\end{array}$ \\
\hline 19 & Survived & $\mathrm{Y}$ & E. coli & $<10^{3}$ & $<10^{3}$ & $<10^{3}$ & \\
\hline 20 & Survived & $\mathrm{N}$ & S. pneumoniae & $\geqslant 10^{3}$ & $\geqslant 10^{3}$ & $\geqslant 10^{3}$ & $\geqslant 10^{3}$ \\
\hline 21 & Survived & $\mathrm{N}$ & P. aeruginosa & $\geqslant 10^{3}$ & $\geqslant 10^{3}$ & & $\geqslant 10^{3}$ \\
\hline 22 & Deceased & $\mathrm{N}$ & $\begin{array}{l}\text { K. pneumoniae } \\
P . \text { aeruginosa }\end{array}$ & $\begin{array}{l}\geqslant 10^{3} \\
\geqslant 10^{3} \\
\geqslant 10^{3}\end{array}$ & $\begin{array}{l}\geqslant 10^{3} \\
\geqslant 10^{3}\end{array}$ & $\begin{array}{l}\geqslant 10^{3} \\
\geqslant 10^{3}\end{array}$ & $\begin{array}{l}\geqslant 10^{3} \\
\geqslant 10^{3}\end{array}$ \\
\hline 23 & Survived & $\mathrm{N}$ & $\begin{array}{c}\text { Streptococcus spp. } \\
\text { S. aureus } \\
\text { Citrobacter spp. }\end{array}$ & $\begin{array}{l}\geqslant 10^{3} \\
\geqslant 10^{3} \\
\geqslant 10^{3}\end{array}$ & $\begin{array}{l}\geqslant 10^{3} \\
\geqslant 10^{3} \\
\geqslant 10^{3}\end{array}$ & $\begin{array}{l}\geqslant 10^{3} \\
\geqslant 10^{3} \\
\geqslant 10^{3}\end{array}$ & $\begin{array}{l}\geqslant 10^{3} \\
\geqslant 10^{3} \\
\geqslant 10^{3}\end{array}$ \\
\hline 24 & Survived & $\mathrm{N}$ & $\begin{array}{l}\text { P. aeruginosa } \\
\text { Streptococcus spp. } \\
\text { Neisseria } \mathrm{spp} .\end{array}$ & $\begin{array}{l}\geqslant 10^{3} \\
\geqslant 10^{3} \\
\geqslant 10^{3}\end{array}$ & $\begin{array}{l}\geqslant 10^{3} \\
\geqslant 10^{3} \\
\geqslant 10^{3}\end{array}$ & $\begin{array}{l}\geqslant 10^{3} \\
\geqslant 10^{3} \\
\geqslant 10^{3}\end{array}$ & $\begin{array}{l}<10^{3} \\
\geqslant 10^{3} \\
<10^{3}\end{array}$ \\
\hline $\begin{array}{l}25 \\
26\end{array}$ & $\begin{array}{l}\text { Survived } \\
\text { Survived }\end{array}$ & $\begin{array}{l}\mathrm{N} \\
\mathrm{N}\end{array}$ & $\begin{array}{l}\text { Streptococcus spp. } \\
\text { P. aeruginosa } \\
\text { A. xylooxydans }\end{array}$ & $\begin{array}{l}\geqslant 10^{3} \\
\geqslant 10^{3} \\
\geqslant 10^{3}\end{array}$ & & $\geqslant 10^{3}$ & \\
\hline $\begin{array}{l}27 \\
28\end{array}$ & $\begin{array}{l}\text { Survived } \\
\text { Survived }\end{array}$ & $\begin{array}{l}\mathrm{Y} \\
\mathrm{N}\end{array}$ & $\begin{array}{l}\text { P. aeruginosa } \\
\text { S. aureus }\end{array}$ & $\begin{array}{l}<10^{3} \\
<10^{3}\end{array}$ & & $<10^{3}$ & $<10^{3}$ \\
\hline 29 & Survived & $\mathrm{N}$ & $\begin{array}{c}\text { P. aeruginosa } \\
\text { S. aureus } \\
\text { Neisseria } \mathrm{spp} . \\
\text { Streptococcus spp. }\end{array}$ & $\begin{array}{l}\geqslant 10^{3} \\
\geqslant 10^{3} \\
\geqslant 10^{3} \\
\geqslant 10^{3}\end{array}$ & $\begin{array}{l}\geqslant 10^{3} \\
\geqslant 10^{3}\end{array}$ & $\begin{array}{l}\geqslant 10^{3} \\
<10^{3} \\
\geqslant 10^{3}\end{array}$ & $\begin{array}{l}\geqslant 10^{3} \\
<10^{3} \\
<10^{3}\end{array}$ \\
\hline 30 & Survived & $\mathrm{N}$ & Streptococcus spp. & & & $\geqslant 10^{3}$ & $\geqslant 10^{3}$ \\
\hline 31 & Survived & $\mathrm{Y}$ & $\begin{array}{l}\text { Proteus spp. } \\
\text { P. aeruginosa } \\
\text { E. coli } \\
\text { S. marcescens } \\
\text { Streptococcus spp. }\end{array}$ & $\begin{array}{l}\geqslant 10^{3} \\
\geqslant 10^{3} \\
\geqslant 10^{3} \\
\geqslant 10^{3} \\
\geqslant 10^{3}\end{array}$ & $\begin{array}{l}\geqslant 10^{3} \\
\geqslant 10^{3} \\
\geqslant 10^{3} \\
\geqslant 10^{3} \\
\geqslant 10^{3}\end{array}$ & $\begin{array}{l}\geqslant 10^{3} \\
\geqslant 10^{3} \\
\geqslant 10^{3} \\
\geqslant 10^{3} \\
\geqslant 10^{3}\end{array}$ & $\begin{array}{l}\geqslant 10^{3} \\
\geqslant 10^{3} \\
\geqslant 10^{3} \\
\geqslant 10^{3} \\
\geqslant 10^{3}\end{array}$ \\
\hline $\begin{array}{l}32 \\
33\end{array}$ & $\begin{array}{l}\text { Survived } \\
\text { Survived }\end{array}$ & $\begin{array}{l}\mathrm{N} \\
\mathrm{N}\end{array}$ & $\begin{array}{l}\text { nil } \\
\text { nil }\end{array}$ & & & & \\
\hline 34 & Survived & $\mathrm{Y}$ & $\begin{array}{c}\text { E. coli } \\
\text { Streptococcus spp. }\end{array}$ & $\begin{array}{l}\geqslant 10^{3} \\
\geqslant 10^{3}\end{array}$ & $\begin{array}{l}\geqslant 10^{3} \\
\geqslant 10^{3}\end{array}$ & $\begin{array}{l}\geqslant 10^{3} \\
\geqslant 10^{3}\end{array}$ & $\begin{array}{l}\geqslant 10^{3} \\
\geqslant 10^{3}\end{array}$ \\
\hline 35 & Survived & $\mathrm{N}$ & $\begin{array}{c}\text { Corynebacteria spp. } \\
\text { S. marcescens } \\
\text { P. aeruginosa } \\
\text { P. aeruginosa }\end{array}$ & $\begin{array}{l}\geqslant 10^{3} \\
\geqslant 10^{3} \\
\geqslant 10^{3}\end{array}$ & $\begin{array}{l}\geqslant 10^{3} \\
\geqslant 10^{3} \\
<10^{3} \\
\geqslant 10^{3}\end{array}$ & $\begin{array}{l}\geqslant 10^{3} \\
\geqslant 10^{3} \\
<10^{3} \\
\geqslant 10^{3}\end{array}$ & $\begin{array}{l}<10^{3} \\
\geqslant 10^{3}\end{array}$ \\
\hline
\end{tabular}


Table 2. Continued

\begin{tabular}{|c|c|c|c|c|c|c|c|}
\hline \multirow{2}{*}{$\begin{array}{l}\text { Patient } \\
\text { No. }\end{array}$} & \multirow[t]{2}{*}{ Clinical outcome } & \multirow{2}{*}{$\begin{array}{c}\text { Prior } \\
\text { antibiotic } \\
\text { treatment }\end{array}$} & \multirow[t]{2}{*}{ Bacterial species } & \multicolumn{2}{|c|}{ PL } & \multicolumn{2}{|c|}{$\mathrm{PB}$} \\
\hline & & & & $1 \mathrm{st}$ & $2 \mathrm{nd}$ & $1 \mathrm{st}$ & $2 \mathrm{nd}$ \\
\hline 37 & Deceased & $\mathrm{N}$ & $\begin{array}{l}\text { P. aeruginosa } \\
\text { Proteus spp. } \\
\text { A. xylooxydans } \\
\text { S. aureus }\end{array}$ & $\begin{array}{l}\geqslant 10^{3} \\
\geqslant 10^{3} \\
\geqslant 10^{3} \\
\geqslant 10^{3}\end{array}$ & $\begin{array}{l}\geqslant 10^{3} \\
\geqslant 10^{3} \\
\geqslant 10^{3} \\
\geqslant 10^{3}\end{array}$ & $\begin{array}{l}\geqslant 10^{3} \\
\geqslant 10^{3} \\
\geqslant 10^{3} \\
\geqslant 10^{3}\end{array}$ & $\begin{array}{l}\geqslant 10^{3} \\
\geqslant 10^{3} \\
\geqslant 10^{3}\end{array}$ \\
\hline $\begin{array}{l}38 \\
39\end{array}$ & $\begin{array}{l}\text { Survived } \\
\text { Survived }\end{array}$ & $\begin{array}{l}\mathrm{Y} \\
\mathrm{Y}\end{array}$ & $\begin{array}{l}\text { Streptococcus spp. } \\
\text { nil }\end{array}$ & $\geqslant 10^{3}$ & $\geqslant 10^{3}$ & $<10^{3}$ & \\
\hline
\end{tabular}

PL: PSB processed in the laboratory; PB: PSB processed outside the laboratory; N: no; Y: yes; P. aeruginosa: Pseudomonas aeruginosa; E. coli: Escherichia coli; S. epidermidis: Staphylococcus epidermidis; S. aureus; Staphylococcus aureus; A. baumanii: Acinetobacter baumanii; H. influenzae: Haemophilus influenzae; S. pneumoniae; Streptococcus pneumoniae; H. parainfluenzae: Haemophilus parainfluenzae; K. pneumoniae: Klebsiella pneumoniae; A. Xylooxydans: Alcaligenes xylooxydans; S. marcescens: Staphylococcus marcescens.

of a total 156 PSB). There was no influence of antibiotics administration on the positivity or negativity of PSB. The clinical outcomes, the existence of a prior antibiotic treatment and the culture results of the four PSB in the 39 patients are presented in table 2. Patients whose PSB specimens did not reach the $10^{3} \mathrm{cfu} \cdot \mathrm{mL}^{-1}$ threshold were not given antibiotics thereafter.

The repeatability of each PSB processing ( $\mathrm{PB}$ and $\mathrm{PL}_{\mathrm{L}}$ is given in tables 3 and 4 . When considering qualitative results (i.e. presence or absence of bacterial colonies without using the $10^{3} \mathrm{cfu} \cdot \mathrm{mL}^{-1}$ threshold), the concordance of PL results was $82.0 \%(K=0.632)$ versus $79.5 \% \quad(K=0.583)$ for $\mathrm{PB}$. MacNemar tests were nonsignificant for both pairs, assessing absence of systematic difference between first and second PSB. However, K-values did not statistically differ between $\mathrm{PB}_{\mathrm{B}}$ and $\mathrm{PL}_{\mathrm{L}}(\mathrm{p}=0.80)$. When considering quantitative results (i.e. using the $10^{3}$ threshold), the concordance for $P_{L}$ results $(89.7 \%, K=0.795)$ was higher than for $\mathrm{PB}_{\mathrm{B}}$ results $(76.9 \%, \mathrm{~K}=0.537)$ but without statistical significance $(p=0.12)$. Thus, a diagnosis of NP was troublesome because of conflicting data in PSB results in $10.3 \%\left(\mathrm{PL}_{\mathrm{L}}\right), 23.1 \%(\mathrm{~PB})$ and $30.8 \%$ (aggregation of $\mathrm{PB}_{\mathrm{B}}$ and $\mathrm{PL}_{\mathrm{L}}$ results) of the cases. Results were similar in patients without tracheostomy $(n=30)$, with $P_{L}$ and $\mathrm{PB}_{\mathrm{B}} \mathrm{K}$-values of 0.862 and 0.528 , respectively $(\mathrm{p}=0.055)$. The order of sampling the pairs of PSB did not influence culture results.

Micro-organisms were found in 91 of the 156 PSB $(58.3 \%)$, and 176 bacterial species were isolated (table 5). In 14 patients at least one PSB culture disclosed more than one micro-organism in concentrations $\geqslant 10^{3} \mathrm{cfu} \cdot \mathrm{mL}^{-1}$. Polymicrobial cultures were more frequent in patients with tracheostomy (seven out of nine patients with tracheostomy versus seven

Table 3. - Repeatability of the two protected specimen brushes (PSB) processed in the laboratory (PL) and outside the laboratory (РB) in 39 episodes of suspected nosocomial pneumonia: qualitative results

\begin{tabular}{|c|c|c|c|c|c|}
\hline \multirow{3}{*}{\multicolumn{2}{|c|}{ Qualitative results }} & \multicolumn{4}{|c|}{ First PSB } \\
\hline & & \multicolumn{2}{|l|}{ PL } & \multicolumn{2}{|l|}{ Рв } \\
\hline & & Recovery of bacteria & Sterile culture & Recovery of bacteria & Sterile culture \\
\hline Second PSB & Recovery of bacteria & 20 & 1 & 18 & 4 \\
\hline \multicolumn{2}{|c|}{$\begin{array}{l}\text { K cofficient } \pm S D \\
\text { Ppos/Pneg }\end{array}$} & $\begin{array}{r}0.632 \pm 0 \\
0.851 / 0.7\end{array}$ & 26 & \multicolumn{2}{|c|}{$0.818 / 0.746$} \\
\hline
\end{tabular}

Ppos: positive agreement; Pneg: negative agreement. $\mathrm{p}=0.8$.

Table 4. - Repeatability of the two protected specimen brushes (PSB) processed in the laboratory $(\mathrm{PL})$ and outside the laboratory $(\mathrm{PB})$ in nosocomial pneumonia: quantitative results

Quantitative results

First PSB

\begin{tabular}{|c|c|c|c|c|c|}
\hline & & \multirow{2}{*}{\multicolumn{2}{|c|}{ PL }} & & \\
\hline & & & & \multicolumn{2}{|c|}{ Рв } \\
\hline & & $\geqslant 10^{3} \mathrm{cfu} \cdot \mathrm{mL}^{-1}$ & $<10^{3} \mathrm{cfu} \cdot \mathrm{mL}^{-1}$ & $\geqslant 10^{3} \mathrm{cfu} \cdot \mathrm{mL}^{-1}$ & $<10^{3} \mathrm{cfu} \cdot \mathrm{mL}^{-1}$ \\
\hline Second PSB & $\begin{array}{l}\geqslant 10^{3} \mathrm{cfu} \cdot \mathrm{mL}^{-1} \\
<10^{3} \mathrm{cfu} \cdot \mathrm{mL}^{-1}\end{array}$ & $\begin{array}{c}18 \\
3\end{array}$ & $\begin{array}{c}1 \\
17\end{array}$ & $\begin{array}{c}14 \\
5\end{array}$ & $\begin{array}{c}4 \\
16\end{array}$ \\
\hline \multicolumn{2}{|c|}{$\begin{array}{l}\text { K coefficient } \pm \mathrm{SD} \\
\mathrm{Ppos} / \mathrm{Pneg}\end{array}$} & \multicolumn{2}{|c|}{$\begin{array}{c}0.795 \pm 0.097 \\
0.900 / 0.895\end{array}$} & \multicolumn{2}{|c|}{$\begin{array}{c}0.537 \pm 0.135 \\
0.757 / 0.780\end{array}$} \\
\hline
\end{tabular}

Ppos: positive agreement; Pneg: negative agreement. $\mathrm{p}=0.12$. 
Table 5.-Micro-organisms isolated from 156 protected specimen brushes performed in 39 episodes of suspected nosocomial pneumonia

\begin{tabular}{|c|c|c|c|c|c|}
\hline \multirow[t]{2}{*}{ Micro-organisms } & \multirow[t]{2}{*}{ Total specimens } & \multicolumn{2}{|c|}{ Monomicrobial } & \multicolumn{2}{|c|}{ Polymicrobial } \\
\hline & & $<10^{3} \mathrm{cfu} \cdot \mathrm{mL}^{-1}$ & $\geqslant 10^{3} \mathrm{cfu} \cdot \mathrm{mL}^{-1}$ & $<10^{3} \mathrm{cfu} \cdot \mathrm{mL}^{-1}$ & $\geqslant 10^{3} \mathrm{cfu} \cdot \mathrm{mL}^{-1}$ \\
\hline Total & 176 & $10(5.7)$ & $26(14.8)$ & $18(10.2)$ & $122(69.3)$ \\
\hline Gramnegative bacteria & $113(64.2)$ & 7 & 14 & 10 & 82 \\
\hline Pseudomonas spp. & 42 & 4 & 10 & 4 & 24 \\
\hline Escherichia coli & 15 & 3 & 0 & 0 & 12 \\
\hline Other Enterobacteriacae & 15 & 0 & 0 & 0 & 15 \\
\hline Moraxella and Neisseria spp. & 15 & 0 & 0 & 5 & 10 \\
\hline Proteus and Providencia spp. & 11 & 0 & 0 & 1 & 10 \\
\hline Acinetobacter and Alcaligenes spp. & 9 & 0 & 0 & 0 & 9 \\
\hline Haemophilus spp. & 6 & 0 & 4 & 0 & 2 \\
\hline Grampositive bacteria & $63(35.8)$ & 3 & 12 & 8 & 40 \\
\hline Streptococcus spp. & 38 & 0 & 10 & 2 & 26 \\
\hline Staphylococcus spp. & 23 & 3 & 2 & 6 & 12 \\
\hline Corynebacteria spp. & 2 & 0 & 0 & 0 & 2 \\
\hline
\end{tabular}

out of 30 without tracheostomy, odds ratio $(\mathrm{OR})=$ 11.5 (2.1-60.9), $\mathrm{p}<0.01)$. Nine PSB (three patients) were positive for fungal species (Candida albicans and Candida glabrata)). All fungi were found in polymicrobial cultures and none were considered pathogenic. The most frequently isolated organisms were Pseudomonas spp. (23.9\%), Enterobacteriaceae (23.3\%), Streptococcus spp. (21.6\%) and Staphylococcus spp. $(13.1 \%)$. When considering only bacteria recovered at a threshold $\geqslant 10^{3} \mathrm{cfu} \cdot \mathrm{mL}^{-1}$, a similar distribution was found: Enterobacteriaceae $(25.0 \%)$, Streptococcus spp. $(24.3 \%)$, P. aeruginosa $(23.0 \%)$ and Staphylococcus spp. $(9.5 \%)$.

The clinical implications in directing the choice of antibiotherapy based on the bacteria recovered at a threshold $\geqslant 10^{3} \mathrm{cfu} \cdot \mathrm{mL}^{-1}$ in at least one of the two PSB were assessed. The diagnosis of NP was hence considered in 22/39 $\mathrm{PL}$ results and 23/39 $\mathrm{PB}_{\mathrm{B}}$ results. Bacteriological discrepancies leading to a troublesome choice in antibiotherapy included species recovered in concentration $\geqslant 10^{3} \mathrm{cfu} \cdot \mathrm{mL}^{-1}$ in one PSB while absent in the other $(17.9 \%$ of the patients with PL results and $23.1 \%$ of the patients with $\mathrm{PB}_{\mathrm{B}}$ results, $\mathrm{p}=\mathrm{NS}$ between
$\mathrm{PL}_{\mathrm{L}}$ and $\mathrm{PB}_{\mathrm{B}}$, and results spread out on each side of the $10^{3} \mathrm{cfu} \cdot \mathrm{mL}^{-1}$ cut-off $\left(2.6 \%\right.$ of the patients with $\mathrm{PL}_{\mathrm{L}}$ results and $15.4 \%$ of the patients with $\mathrm{PB}_{\mathrm{B}}$ results, p-value was not significant between $P_{L}$ and $P_{B}$ ). Table 6 summarizes the bacteriological discrepancies in patients in whom a diagnosis of NP was considered.

\section{Discussion}

In contrast with VAP for which the literature is abundant [22], the management of NP in nonintubated patients remains poorly documented [5]. The diagnosis of NP in nonintubated patients is difficult because, as in VAP, it may be assumed that its clinical (i.e. noninvasive) diagnosis is imprecise and less accurate than with invasive methods [14]. In addition, the validation of a reliable technique of microbiological diagnosis of NP, with a gold standard of diagnosis, such as histological confirmation of pneumonia [12], is unlikely to be done in most of the nonintensive care units of medical and surgical wards. However, in selected centres, NP diagnosis strategy in

Table 6. - Bacteriological discrepancies in patients in whom a diagnosis of nosocomial pneumonia was considered (at least one species isolated from at least one protected specimen brush (PSB) in concentration $\geqslant 10^{3} \mathrm{cfu} \cdot \mathrm{mL}^{-1}$ )

\begin{tabular}{|c|c|c|c|c|}
\hline & \multicolumn{2}{|c|}{$\begin{array}{l}\text { Species recovered in concentration } \\
\geqslant 10^{3} \mathrm{cfu} \cdot \mathrm{mL}^{-1} \text { in one PSB while absent in the other }\end{array}$} & \multicolumn{2}{|c|}{$\begin{array}{l}\text { Samples spread out on each } \\
\text { side of the } 10^{3} \mathrm{cfu} \cdot \mathrm{mL}^{-1} \text { cut-off }\end{array}$} \\
\hline & PL & $\mathrm{PB}$ & PL & $\mathrm{PB}$ \\
\hline Patients & 7 & 9 & 1 & 6 \\
\hline Species & 11 & 12 & 1 & 6 \\
\hline Gramnegative bacteria & 3 & 5 & 1 & 5 \\
\hline Pseudomonas spp. & 1 & 2 & 1 & 2 \\
\hline Enterobacteriacae & 0 & 2 & 0 & 1 \\
\hline Moraxella and Neisseria spp. & 1 & 1 & 0 & 2 \\
\hline Alcaligenes spp. & 1 & 0 & 0 & 0 \\
\hline Grampositive bacteria & 8 & 7 & 0 & 1 \\
\hline Streptococcus spp. & 5 & 5 & 0 & 0 \\
\hline Staphylococcus spp. & 2 & 1 & 0 & 1 \\
\hline Corynebacteria spp. & 1 & 1 & 0 & 0 \\
\hline
\end{tabular}

PL: PSB processed in the laboratory; PB: PSB processed outside the laboratory. 
nonventilated patients is built on fibreoptic bronchoscopy with lower respiratory secretion specimens obtained either by PSB or BAL.

The present study was designed to assess the repeatability of PSB in nonventilated patients. This issue has been largely addressed in VAP, but to the authors' knowledge, has not been studied in nonventilated patients. As in VAP, the repeatability of the PSB may also be questioned in nonventilated patients. It was found that the results of PSB are dependent on sampling processing, as shown by higher repeatability when the PSB are transected inside rather than outside the laboratory. The difference was not statistically significant, but due to the low number of patients included, statistical testing power was low. These preliminary data may justify additional studies on a larger scale.

To the authors' knowledge, three studies addressed the repeatability of PSB or BAL in VAP. MARQUETTE et al. [13] found a $13.6 \%$ discordance in quantitative bacterial results, but there was a noticeable intrasubject variability $(16 \%)$. TIMSIT et al. [11] found similar results with a $24 \%$ discordance with regard to the recommended diagnostic threshold of $10^{3} \mathrm{cfu} \cdot \mathrm{mL}^{-1}$. The repeatability of BAL, with pulmonary infection confirmed above a $10^{4} \mathrm{cfu} \cdot \mathrm{mL}^{-1}$ threshold was studied by Gerbeaux et al. [23]. The $\mathrm{K}$-value was 0.74 in this instance, with a $9 \%$ discordance of BAL samplings. The presented results (a discordance rate of 10.3\% for laboratory processing of PSB and 23.1\% for bedside processing of PSB) are similar to the discordance rates found by MARQUETTE et al. [13] (13.6\% with laboratory transection) and TIMSIT et al. [11] ( $24 \%$ with bedside transection). In addition, because of conflicting data in the bacteriological results, the antibiotic choice when the diagnosis of NP was considered would have been troublesome in $31.8 \%$ $(7 / 22)$ of the patients after laboratory processing of PSB and $56.5 \%(13 / 23)$ of the patients after bedside processing of PSB. This finding confirms that in nonintensive care unit patients, as reported in VAP $[11,24]$, it is difficult to choose an adequate treatment of NP because of the low degree of repeatability of this technique.

The patients were all studied in a secondary care facility, and most of them had previously stayed in another hospital, so every potential pulmonary infectious manifestation was attributable to NP rather than community-acquired pneumonia. In addition, all patients had at least one risk factor predisposing to NP [21]. The distribution of the recovered pathogens show that the most frequently isolated organisms were Pseudomonas spp. (23.9\%), Enterobacteriaceae (23.3\%), Streptococcus spp. (21.6\%) and Staphylococcus spp. (13.1\%). The distribution of the microorganisms of the present study is similar to that reported by TROUILLET et al. [25] in a study performed in a Parisian intensive care unit, including the isolation of fungal agents.

In conclusion, as for ventilator-associated pneumonia, there is a low degree of repeatability of protected specimen brush outside intensive care units. Since the results of protected specimen brush seem to be dependent on sampling procedures, it is suggested that protected specimen brush should be processed in nonintubated patients with as little manipulation as possible and, when possible, all processing should be exclusively performed inside the laboratory. Further studies are needed to improve the diagnostic procedure of nosocomial pneumonia in nonintensive care unit patients, since the distribution of pathogens found in cases of suspected of nosocomial pneumonia in nonventilated patients appears to be similar to that obtained in ventilator-associated pneumonia.

\section{References}

1. Enquête nationale de prévalence des infections nosocomiales. BEH 1996; 36: $161-163$.

2. American Thoracic Society. Hospital-acquired pneumonia in adults: diagnosis, assessment of severity, initial antimicrobial therapy, and preventive strategies. A consensus statement, American Thoracic Society, November 1995. Am J Respir Crit Care Med 1996; 153: $1711-1725$.

3. Fagon JY, Chastre J, Hance AJ, Montravers P, Novara A, Gibert C. Nosocomial pneumonia in ventilated patients: a cohort study evaluating attributable mortality and hospital stay. Am J Med 1993; 94: $281-288$.

4. Heyland DK, Cook DJ, Griffith L, Keenan SP, BrunBuisson C. The attributable morbidity and mortality of ventilator-associated pneumonia in the critically ill patient. The Canadian Critical Trials Group. Am J Respir Crit Care Med 1999; 159: 1249-1256.

5. Gomez J, Esquinas A, Agudo MD, et al. Retrospective analysis of risk factors and prognosis in nonventilated patients with nosocomial pneumonia. Eur J Clin Microbiol Infect Dis 1995; 14: 176-181.

6. Chastre J, Fagon JY. Invasive diagnostic testing should be routinely used to manage ventilated patients with suspected pneumonia. Am J Respir Crit Care Med 1994; 150: 570-574.

7. Niederman MS, Torres A, Summer W. Invasive diagnostic testing is not needed routinely to manage suspected ventilator-associated pneumonia. Am J Respir Crit Care Med 1994; 150: 565-569.

8. Wimberley NW, Bass JB Jr, Boyd BW, et al. Use of a bronchoscopic protected catheter brush for the diagnosis of pulmonary infections. Chest 1982; 81: $556-562$.

9. Villers D, Derriennic M, Raffi F, et al. Reliability of the bronchoscopic protected catheter brush in intubated and ventilated patients. Chest 1985; 88: $527-$ 530.

10. Baughman RP, Thorpe JE, Staneck J, Rashkin M, Frame PT. Use of the protected specimen brush in patients with endotracheal or tracheostomy tubes. Chest 1987; 91: 233-236.

11. Timsit JF, Misset B, Francoual S, Goldstein FW, Vaury $\mathrm{P}$, Carlet $\mathrm{J}$. Is protected specimen brush a reproducible method to diagnose ICU-acquired pneumonia? Chest 1993; 104: 104-108.

12. Chastre J, Viau F, Brun P, et al. Prospective evaluation of the protected specimen brush for the diagnosis of pulmonary infections in ventilated patients. Am Rev Respir Dis 1984; 130: 924-929.

13. Marquette $\mathrm{CH}$, Herengt $\mathrm{F}$, Mathieu $\mathrm{D}$, et al. Diagnosis of pneumonia in mechanically ventilated 
patients. Repeatability of the protected specimen brush. Am Rev Respir Dis 1993; 147: 211-214.

14. Fagon JY, Chastre J, Hance AJ, Domart Y, Trouillet JL, Gibert C. Evaluation of clinical judgment in the identification and treatment of nosocomial pneumonia in ventilated patients. Chest 1993; 103: 547-553.

15. Tobin MJ, Grenvik A. Nosocomial lung infection and its diagnosis. Crit Care Med 1984; 12: 191-201.

16. Baselski VS, el-Torky M, Coalson JJ, Griffin JP. The standardization of criteria for processing and interpreting laboratory specimens in patients with suspected ventilator-associated pneumonia. Chest 1992; 102: $571 \mathrm{~S}-579 \mathrm{~S}$.

17. Cohen J. A coefficient of agreement for nominal scales. Educ Psychol Measmt 1960; 20: 37-46.

18. Feinstein AR, Cicchetti DV. High agreement but low kappa: I. The problems of two paradoxes. J Clin Epidemiol 1990; 43: 543-549.

19. Cicchetti DV, Feinstein AR. High agreement but low kappa: II. Resolving the paradoxes. J Clin Epidemiol 1990; 43: $551-558$.

20. Fermanian J. Mesure de l'accord entre deux juges cas qualitatif. Rev Epidemiol Sante Publique 1984; 32: $140-147$.

21. Celis R, Torres A, Gatell JM, Almela M, RodriguezRoisin R, Agusti-Vidal A. Nosocomial pneumonia. A multivariate analysis of risk and prognosis. Chest 1988; 93: 318-324.

22. Wunderink RG. Mortality and the diagnosis of ventilator-associated pneumonia: a new direction. Am J Respir Crit Care Med 1998; 157: 349-350.

23. Gerbeaux P, Ledoray V, Boussuges A, Molenat F, Jean P, Sainty JM. Diagnosis of nosocomial pneumonia in mechanically ventilated patients: repeatability of the bronchoalveolar lavage. Am J Respir Crit Care Med 1998; 157: 76-80.

24. Dreyfuss D, Mier L, Le Bourdelles G, et al. Clinical significance of borderline quantitative protected specimen brush results. Am Rev Respir Dis 1993; 147: 946 951.

25. Trouillet JL, Chastre J, Vuagnat A, et al. Ventilatorassociated pneumonia caused by potentially drugresistant bacteria. Am J Respir Crit Care Med 1998; 157: $531-539$. 